\title{
USO DE APLICATIVOS EM SMARTPHONE PARA MEDIÇÕES DE ÁRVORES
}

\author{
USE OF SMARTPHONE APLICATIONS FOR TREES MEASUREMENTS
}

\author{
Tieme Breternitz Harfouche ${ }^{1}$, Ana Paula Dalla Corte ${ }^{2}$, Marieli Ruza ${ }^{3}$, Alexandre Behling ${ }^{4}$ \\ 1,2,3,4 Universidade Federal do Paraná, Curitiba, Paraná, Brasil - tiemebretzh@gmail.com, \\ anapaulacorte@gmail.com, marielisabrina93@gmail.com \& alexandre.behling@yahoo.com.br
}

\section{RESUMO}

Este trabalho visou verificar a precisão de aplicativos de smartphone para medir indiretamente a altura de árvores e a distância entre o operador e a árvore. Como testemunha para medir altura, adotou-se o Hipsômetro Vertex IV (TOa) e, para distância, a variável medida com trena (TOb). Foram selecionadas 30 árvores isoladas do Campus da Universidade Federal do Paraná em Curitiba - PR e 30 árvores pertencentes à um plantio de Eucalyptus sp. na Fazenda Experimental Canguiri em Pinhais - PR. Foram 7 tratamentos testados para altura: Smart Measure (T1a), sMeasure (T2a), Distance meter (T3a), Hypsometer (T4a), 3D-Prumo (T5a), Height and Distance (T6a) e Tree-H (T7a). Foram 8 tratamentos para medir distância: Smart Measure (T1b), sMeasure (T2b), Distance meter (T3b), Hypsometer (T4b), 3D-Prumo (T5b), Height and Distance (T6b), Easy Measure (T7b) e Smart Distance (T8b). Avaliou-se a precisão dos aplicativos com o teste $t$ de Student pareado com $5 \%$ de significância em relação às testemunhas e cálculo dos erros. Para altura em árvores isoladas, o tratamento T2a apresentou diferença estatística a probabilidade de $5 \%$. 0 tratamento T6a obteve menor erro $(2,48 \mathrm{~m})$. No plantio de Eucalyptus sp., os tratamentos T1a, T6a e T7a apresentaram diferenças significativas e T3a obteve menor erro $(2,99 \mathrm{~m})$. Para a medição de distância em árvores isoladas, os tratamentos T4b e T6b não apresentaram diferença e T4b apresentou menor erro (2,28 m). No plantio, todos apresentaram diferença estatística. A incorporação de aplicativos para smartphones é alternativa para uso em inventários florestais para alturas. Para distâncias, deve-se ter cautela.

PALAVRAS-CHAVE: Altura total, Distância, Inventário florestal, Tecnologia.

\begin{abstract}
This work aimed to verify the smartphone applications' accuracy to indirectly measure trees' height and the distance between the operator and the tree. As a control to measure height, the Vertex IV hypsometer (TOa) was adopted and, for distance, the variable measured with scale (TOb). 30 trees isolated were selected from Campus of Federal University of Paraná (Curitiba - PR) and 30 trees belonging to a planting of Eucalyptus sp. at Canguiri Experimental Farm (Pinhais - PR). Seven treatments were tested for height: Smart Measure (T1a), sMeasure (T2a), Distance meter (T3a), Hypsometer (T4a), 3D-Prumo (T5a), Height and Distance (T6a) and Tree-H. Eight treatments for distance measurement: Smart Measure (T1b), sMeasure (T2b), Distance meter (T3b), Hypsometer (T4b), 3D-Prumo (T5b), Height and Distance (T6b), Easy Measure) and Smart Distance (T8b). The applications' accuracy was evaluated using the paired Student's t-test with $5 \%$ significance in relation to the controls, as well as errors' calculation. For height in isolated trees, the treatment T2a presented a statistical difference of 5\% probability. The treatment T6a obtained the smallest error $(2.48 \mathrm{~m})$. In the Eucalyptus sp. planting, the treatments T1a, T6a and T7a presented significant differences and T3a obtained the lowest error $(2.99 \mathrm{~m})$. For distance measurement in isolated trees, the treatments T4b and T6b presented no difference and T4b presented smallest error $(2.28 \mathrm{~m})$. At planting, all presented statistical difference. The incorporation of smartphones' applications is an alternative for use in forest inventories for heights. For distances, still with caution.
\end{abstract}

KEYWORDS: Total height, Distance, Forest inventory, Technology. 


\section{INTRODUÇÃO}

O Brasil é o segundo país com maior área florestal do mundo. Seu território é composto em $58 \%$ por cobertura florestal plantada e natural (FAO, 2015), sendo que 7,84 milhões de hectares são reflorestamentos (IBÁ, 2017). Por ser um país florestal, a demanda de inventários florestais tem sido crescente, já que estes são base para atividades de manejo, conservação, planejamentos e decisões administrativas (TOMPPO et al., 2010).

Busca-se na execução de inventários florestais, baseados em técnicas de amostragem, métodos que apresentam menor erro e custo, os quais estão diretamente ligados ao tempo de medição de suas variáveis. Portanto, para resultados mais precisos e menos dispendiosos, devem ser adotados métodos adequados às características da população estudada (CESARO et al., 1994).

A determinação da altura de árvores é fundamental na realização de inventários florestais, essa variável é mensurada em parcelas subsidiando o ajuste de uma relação hipsométrica (ARAÚJO et al., 2012), a qual estima alturas através da relação entre o diâmetro da altura do peito (DAP a 1,3 m) e altura total das árvores (THIERSCH et al., 2013).

A altura total também é input para funções de afilamento, as quais estimam diâmetros a diferentes alturas (CAMPOS \& LEITE, 2009). A importância de sua estimativa ou medição está vinculada ao cálculo de volume dos indivíduos de uma floresta, incrementos em altura, além de servir de indicativo para qualidade produtiva de um local, denominado índice de sítio (SILVA et al., 2012).

Os procedimentos para medir-se altura são definidos como diretos através de vara, trena, ou indiretos a partir de aparelhos chamados de hipsômetros, sendo estes fundamentados em métodos geométricos e trigonométricos (SANQUETTA et al., 2014).

Além das alturas, a medição de distâncias horizontais também é corriqueira nos inventários, para a instalação de parcelas, posicionamento de árvores em croqui, com o uso de medidores eletrônicos ou corda, bem como equipamentos como Vertex IV, alcançando distâncias de até 30 metros (SANQUETTA et al., 2014).

Com a presente inovação tecnológica tem sido crescente o uso de smartphones acompanhado de uma gama de aplicativos desenvolvidos para diversas finalidades, incluindo estimativas de alturas e distâncias, tornando-se também uma alternativa que visa à redução de custos em inventários florestais, a partir de evidências de sua eficácia (URBANO et al., 2014). Contudo, não se observam estudos sobre a precisão na coleta de tais variáveis com esses equipamentos.

O trabalho teve como objetivo verificar a precisão de diferentes aplicativos para smartphone desenvolvidos para medir indiretamente a altura das árvores. Também, fez parte do escopo deste trabalho o teste de aplicativos desenvolvidos para medir a distância entre o operador e a árvore.

\section{MATERIAL E MÉTODOS}

\section{Área de estudo}

Foram selecionadas 30 árvores isoladas para realizar os testes, sendo que essas se localizam no Campus Centro Politécnico da Universidade Federal do Paraná, município de Curitiba (PR) (Figura 1). Adicionalmente, também foram selecionadas 30 árvores pertencentes a um plantio de Eucalyptus sp. localizadas na Fazenda Experimental Canguiri, município de Pinhais (PR), região metropolitana de Curitiba (PR) (Figura 2).

O sistema climático de classificação de Köppen, designa a região de Curitiba e de Pinhais pelo tipo climático $\mathrm{Cfb}$, apresentando um clima temperado ou subtropical, úmido mesotérmico (KÖPPEN \& GEIGER, 1928). A temperatura média anual de Curitiba é de $17,4{ }^{\circ} \mathrm{C}$ (EMBRAPA, 2012) e sua altitude de 934,6 m (MARTINI et al., 2013).

O município de Pinhais possui uma temperatura média durante o ano de $16,5^{\circ} \mathrm{C}$ (EMBRAPA, 2012) e altitude média de $900 \mathrm{~m}$, situada no Primeiro Planalto do Paraná (GOMIDE \& LINGNAU, 2009). 

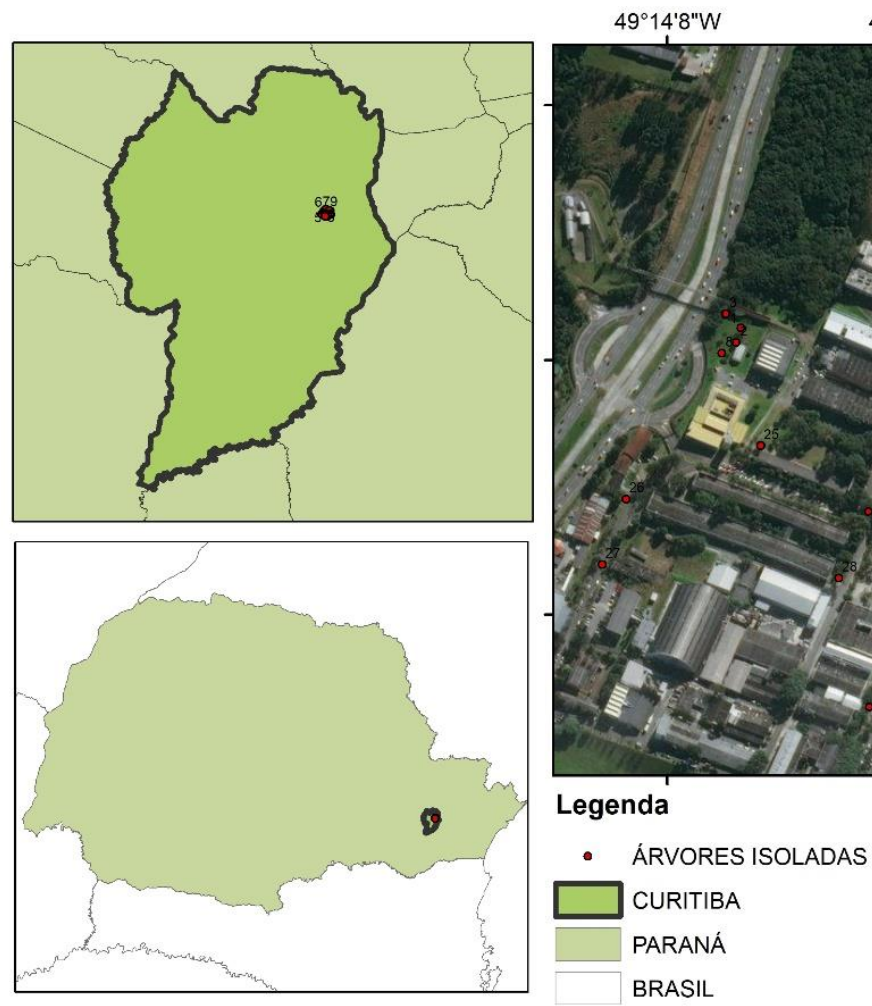

$49^{\circ} 14^{\prime} \mathrm{O}^{\prime \prime} \mathrm{W}$

$49^{\circ} 13^{\prime} 52^{\prime \prime} \mathrm{W}$

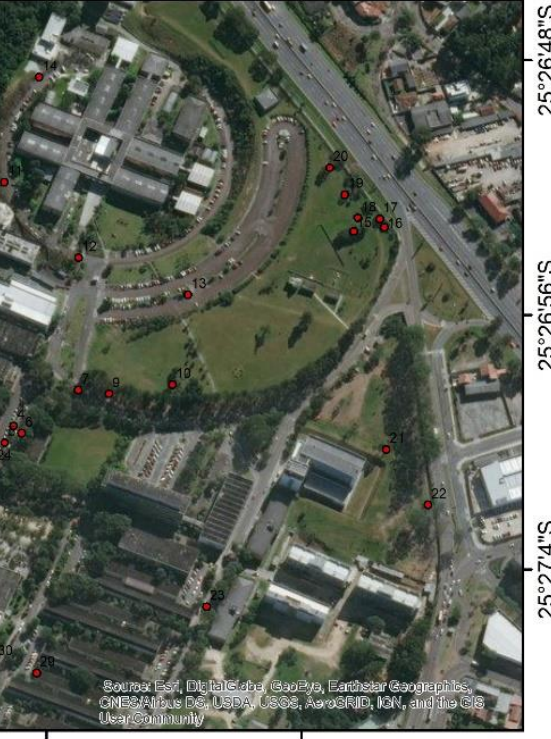

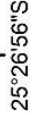

Figura 1. Localização das 30 árvores isoladas no campus Centro Politécnico da UFPR.
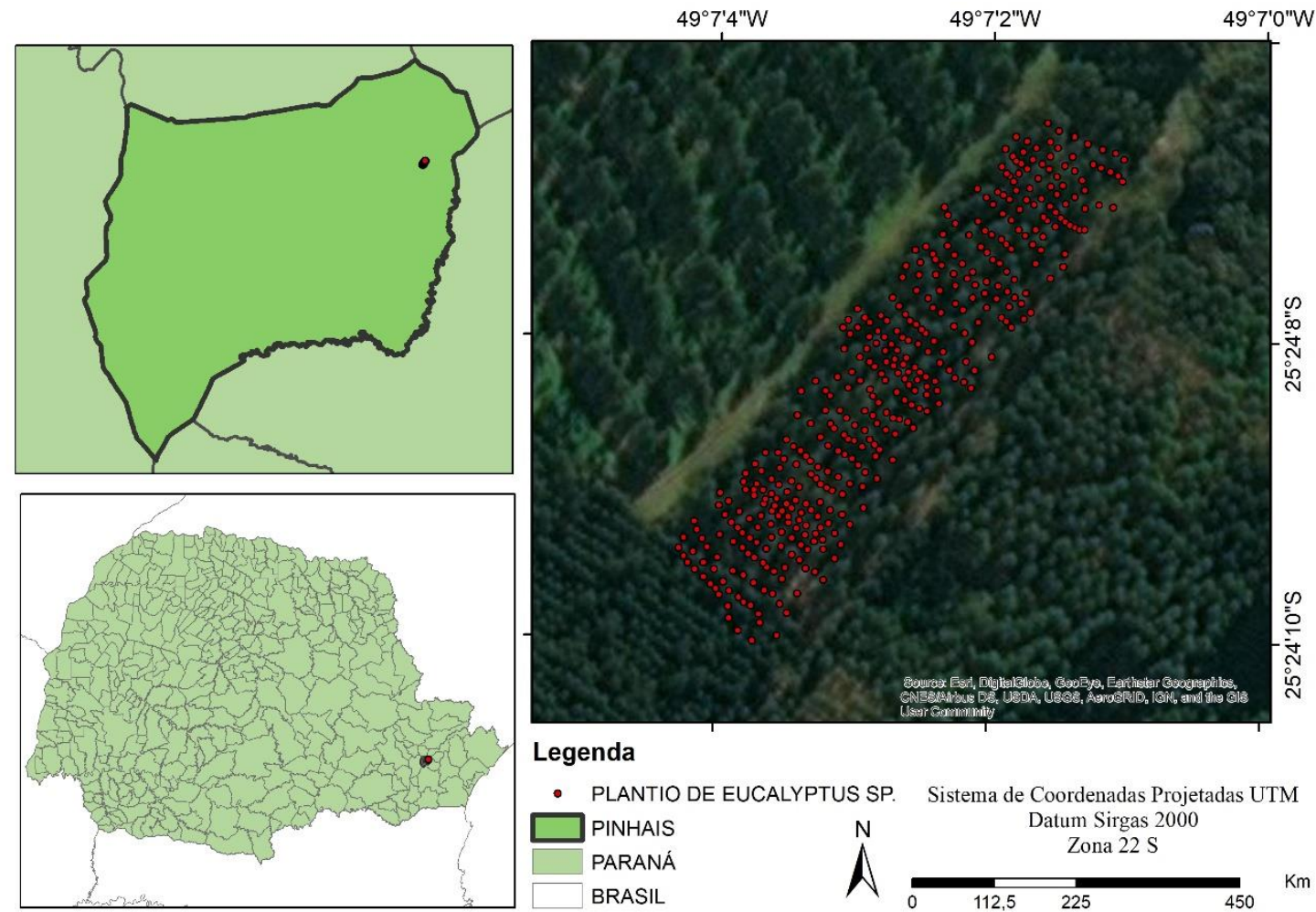

Legenda

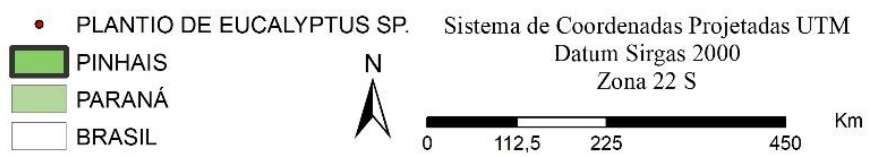

Figura 2. Localização do plantio de Eucalyptus sp. na Fazenda Experimental Canguiri da UFPR. 


\section{Coleta de dados}

Foram medidas alturas e distâncias de 30 árvores isoladas de diferentes espécies (Tabela 1), e 30 árvores de um plantio florestal (linha), tomando como testemunha variáveis reais mensuradas com o auxílio do Vertex IV (TOa) e da trena (TOb), adotando uma distância entre o operador e a árvore de $10 \mathrm{~m}$ em árvores isoladas e $15 \mathrm{~m}$ no plantio em linha. A circunferência a altura do peito (CAP) também foi medida com fita métrica.

Tabela 1. Espécies identificadas das árvores isoladas utilizadas para os testes de mensuração de altura e distância.

\begin{tabular}{|c|c|c|c|}
\hline Árvore & Espécie & Nome popular & $\operatorname{DAP}(\mathrm{cm})$ \\
\hline 1 & Anadenanthera colubrina (Vell.) Brenan & Angico-branco & 18,7 \\
\hline 2 & Melaleuca leucadendra (L.) L. & Melaleuca & 40,1 \\
\hline 3 & Ligustrum lucidum W. T. Aiton & Alfeneiro & 22,6 \\
\hline 4 & Jacaranda mimosifolia D. Don & Jacaranda-mimoso & 22,5 \\
\hline 5 & Magnolia grandiflora L. & Magnolia-branca & 19,3 \\
\hline 6 & Tipuana tipu (Benth.) Kuntze & Tipuana & 35,7 \\
\hline 7 & Ligustrum lucidum W. T. Aiton & Alfeneiro & 29,3 \\
\hline 8 & Liquidambar styraciflua $\mathrm{L}$. & Liquidambar & 33,1 \\
\hline 9 & Eucalyptus sp. & Eucalipto & 65,9 \\
\hline 10 & Magnolia grandiflora $\mathrm{L}$. & Magnolia-branca & 39,8 \\
\hline 11 & Platanus $x$ acerifolia (Aiton) Willd. & Plátano & 24,2 \\
\hline 12 & Platanus $x$ acerifolia (Aiton) Willd. & Plátano & 38,5 \\
\hline 13 & Magnolia grandiflora L. & Magnolia-branca & 41,1 \\
\hline 14 & Platanus $x$ acerifolia (Aiton) Willd. & Plátano & 26,6 \\
\hline 15 & Ligustrum lucidum W. T. Aiton & Alfeneiro & 43,3 \\
\hline 16 & Cassia leptophylla Vogel & Falso-barbatimão & 29,6 \\
\hline 17 & Jacaranda mimosifolia D. Don & Jacaranda-mimoso & 28 \\
\hline 18 & Lafoensia pacari A. St. - Hill. & Dedaleiro & 18,1 \\
\hline 19 & Lafoensia pacari A. St. - Hill. & Dedaleiro & 31,8 \\
\hline 20 & Corymbia eximia (Schauer) K. D. Hill \& L.A.S. Johnson & Eucalipto & 35,2 \\
\hline 21 & Eucalyptus sp. & Eucalipto & 28,5 \\
\hline 22 & Peltophorum dubium (Spreng.) Taub. & Canafístula & 36,9 \\
\hline 23 & Lagerstroemia indica $\mathrm{L}$. & Extremosa & 38,5 \\
\hline 24 & Lagerstroemia indica L. & Extremosa & 29,0 \\
\hline 25 & Tipuana tipu (Benth.) Kuntze & Tipuana & 32,8 \\
\hline 26 & Ceiba speciosa A. St. - Hill & Paineira & 69,4 \\
\hline 27 & Liquidambar styraciflua $\mathrm{L}$. & Liquidambar & 43,0 \\
\hline 28 & Eucalyptus sp. & Eucalipto & 71,6 \\
\hline 29 & Casearia decandra Jacq. & Guaçatunga & 30,9 \\
\hline 30 & Ligustrum lucidum W. T. Aiton & Alfeneiro & 37,6 \\
\hline
\end{tabular}

Os aplicativos testados para as medições e usados para a comparação com a testemunha foram instalados em um
Smartphone Samsung modelo Galaxy S5 para os testes, descritos no Quadro 1. 
Quadro 1. Tratamentos avaliados na medição de altura e distância em árvores isoladas e plantio em linha, considerando 7 métodos para altura e 8 para distância.

\begin{tabular}{|c|c|c|c|}
\hline Método & Tratamento para altura & Tratamento para distância & Descrição \\
\hline Smart Measure & T1a & $\mathrm{T} 1 \mathrm{~b}$ & Mede distância e altura de um alvo por trigonometria \\
\hline sMeasure & T2a & $\mathrm{T} 2 \mathrm{~b}$ & $\begin{array}{l}\text { Mede distância e a altura de um alvo por trigonometria } \\
\text { simples, pode ser usado por qualquer Smartphone }\end{array}$ \\
\hline Distance meter & T3a & T3b & $\begin{array}{l}\text { Calcula a distância e altura aproximada de um objeto } \\
\text { utilizando algoritmo matemático }\end{array}$ \\
\hline Hypsometer & T4a & T4b & $\begin{array}{l}\text { Mede a distância e altura de árvores tendo por base a } \\
\text { altura da câmera em relação ao chão }\end{array}$ \\
\hline 3D-Prumo & T5a & $\mathrm{T} 5 \mathrm{~b}$ & Dentre outras funções, mede altura e distância \\
\hline Height and Distance & T6a & T6b & Mede a distância e altura do objeto em relação ao operador \\
\hline EasyMeasure & - & $\mathrm{T} 7 \mathrm{~b}$ & $\begin{array}{l}\text { Mostra a distância até os objetos vistos através da lente da } \\
\text { câmera de um telefone ou tablet }\end{array}$ \\
\hline Smart Distance & - & T8b & $\begin{array}{l}\text { Mede a distância a um alvo com a câmera, conhecendo-se a } \\
\text { altura do alvo, com uma distância efetiva de } 10 \mathrm{~m}-1 \mathrm{~km}\end{array}$ \\
\hline Tree-H & T7a & - & Mede a altura de árvores por método geométrico \\
\hline
\end{tabular}

Uma vez obtidos os dados de altura e distância dos tratamentos e testemunhas, verificou-se a normalidade das amostras por meio do teste de normalidade Kolmogorov-Smirnov. Em seguida, para a avaliar a precisão dos aplicativos utilizou-se o teste $t$ a $5 \%$ de probabilidade, com duas amostras em par para as médias, para amostras dependentes, em relação às testemunhas (altura, distância), bem como, o cálculo dos erros para cada método de estimação (Quadro 2). Utilizou-se a média dos erros (Erro \%) das amostras para cada tratamento, sendo apresentados nas Tabelas 3 e 5.

Quadro 2. Medidas estatísticas padrão utilizadas para o cálculo e análise de erros para avaliação dos métodos de medição de altura total e distância.

Erro médio (ME)

Erro quadrático médio (MSE)

Raiz do erro quadrático médio (RMSE)

Erro quadrático médio relativo (RRMSE)

Erro (\%)

$$
\begin{gathered}
M E=\frac{\sum_{t=1}^{n} d i}{n} \\
M S E=\frac{\sum_{t=i}^{n} d i^{2}}{n} \\
R M S E=\sqrt{\frac{\sum_{t=i}^{n} d i^{2}}{n}} \\
R R M S E=\left(\frac{\sqrt{\frac{\sum_{t=i}^{n} d i^{2}}{n}}}{\bar{y}}\right) * 100 \\
E(\%)=\left(\frac{y_{o b s}-y_{e s t}}{y_{o b s}}\right) * 100
\end{gathered}
$$

$d i=$ valor observado - valor estimado; $\mathrm{n}=$ número de amostras; $\bar{y}=$ média dos valores observados; $y_{o b s}=$ valor observado; e $y_{e s t}=$ valor estimado. 


\section{RESULTADOS E DISCUSSÃO}

Os resultados mostraram que apenas o tratamento $\mathrm{T} 2 \mathrm{a}$, testado para altura em árvores isoladas, apresentou diferença estatística à probabilidade de $5 \%$. No plantio de Eucalyptus sp., os tratamentos T1a, T6a e T7a apresentaram diferenças significativas a $5 \%$. Vale ressaltar que os tratamentos T3a, T4a e T5a, portanto, não mostraram diferença estatística em relação à testemunha tanto em árvores isoladas quanto na área de plantio. 0 tratamento T7a apresentou limitações e dificuldades de operação em ambas as situações, não permitindo a obtenção de resultado em árvores isoladas (Tabela 2).

Tabela 2. Teste $t$ de Student com $5 \%$ de significância em relação a testemunha altura (TOa).

\begin{tabular}{|c|c|c|c|}
\hline \multirow{2}{*}{ Tratamento } & \multirow{2}{*}{ Método } & \multicolumn{2}{|c|}{ Teste $\mathbf{t}$} \\
\hline & & Árvores isoladas & Plantio de Eucalyptus sp. \\
\hline T1a & Smart Measure & $-0,72^{\text {ns }}$ & $2,2^{*}$ \\
\hline T2a & sMeasure & $-2,84 * *$ & $-1,88^{\text {ns }}$ \\
\hline T3a & Distance meter & $-1,51^{\text {ns }}$ & $1,89^{\text {ns }}$ \\
\hline T4a & Hypsometer & $-0,39^{n s}$ & $1,13^{\text {ns }}$ \\
\hline $\mathrm{T} 5 \mathrm{a}$ & 3D-Prumo & $-1,19^{\text {ns }}$ & $1,98^{\mathrm{ns}}$ \\
\hline T6a & Height and Distance & $-0,41^{\text {ns }}$ & $5,08 * *$ \\
\hline T7a & Tree-H & - & $5,9 * *$ \\
\hline
\end{tabular}

$\mathrm{ns}=$ não significativo com $95 \%$ de probabilidade; * = coeficiente significativo a $5 \%$ de probabilidade $(p<0,05)$; e ** = coeficiente significativo a $1 \%$ de probabilidade $(p<0,01)$

Dentre os tratamentos que não apresentaram diferença estatística em suas médias em árvores isoladas, o tratamento T6a obteve um menor erro (RMSE) de 2,48 $\mathrm{m}$, e logo em seguida o tratamento T1a com 2,95 m de erro (RMSE). Dentre os métodos testados no plantio em linha, o tratamento T3a obteve menor $\operatorname{RMSE}(2,99 \mathrm{~m})$ como mostra a Tabela 3.
Segundo o Instituto de Florestas do Paraná - IFPR (2016), em seu Manual de Inventário Florestal, o erro máximo para estimativa de altura para que um trabalho de campo seja aceito é de $10 \%$. Exceto pelo tratamento T2a em árvores isoladas, e os tratamentos T6a e T7a no plantio em linha, os demais tratamentos demostraram em média, um Erro (\%) menor que $10 \%$.

Tabela 3. Análise de erros para diferentes métodos na medição de altura em árvores isoladas e plantio de Eucalyptus sp.

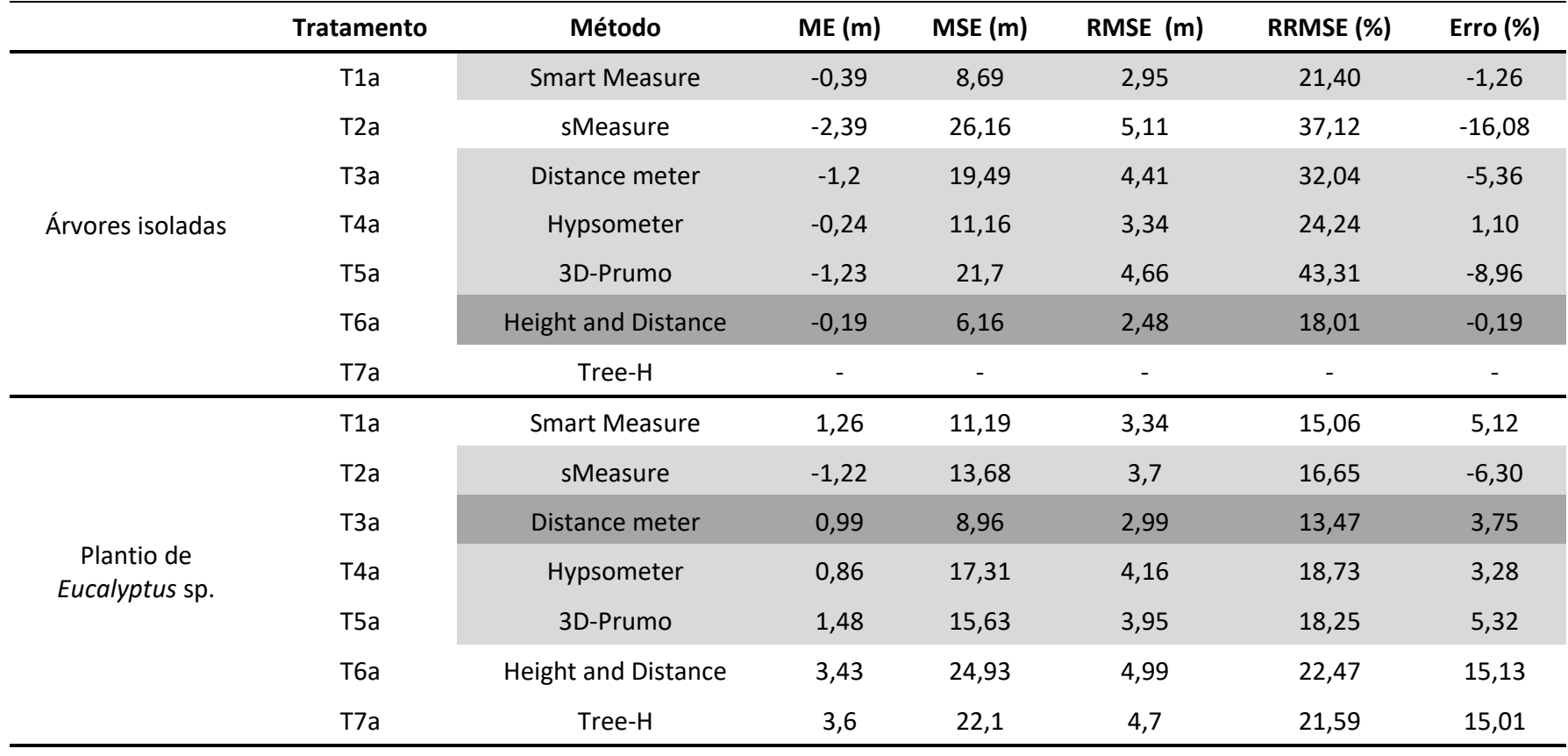


Brito Neto et al. (2016) realizaram um estudo testando o aplicativo telemóvel Smart Measure versão 1.6 para altura total de árvores em povoamentos florestais de eucaliptos em terrenos planos, o qual apresentou erro em porcentagem (E\%) de 2,37\% em relação a altura real, mostrando um resultado satisfatório nessas condições. Contudo, neste trabalho, diferentemente do citado, este mesmo aplicativo apresentou um erro de 5,12\% e diferença estatística no plantio de Eucalyptus sp., sendo eficaz apenas em árvores isoladas.

O estudo realizado por Bijak \& Sarzynski (2015), no qual os aplicativos Smart Measure 2.3 e Measure Height foram testados para altura em um complexo florestal de Pinus sylvestris L. em idade de 25, 52 e 110 anos na Polônia apresentou para o primeiro método um RMSE igual a 1,11 e 1,01 m para 15 e 20 m de distância de medição, enquanto para o software Mesure Height foi maior (2,46 e 2,04 m), respectivamente. $\mathrm{O}$ desempenho do Smart Measure no estudo citado foi melhor do que em ambas situações do trabalho aqui apresentado.

Vastaranta et al. (2015) avaliaram o aplicativo TRESTIMA $^{\text {TM }}$ em uma floresta na Finlândia cuja predominância eram árvores das espécies de Pinus sylvestris L. (pinheiro-silvestre) e Picea Abies (L.) H. Karst. (abeto norueguês). O aplicativo, através de imagens coletadas por meio de smartphone, estima a área basal, diâmetro e altura. Em seus resultados para esta última variável, apresentou 2,1 m e 2,3 m de RMSE em pinheirosilvestre e abeto norueguês, respectivamente, logrando melhor desempenho em comparação aos métodos aqui avaliados para medição de altura.

Considerando inventários florestais realizados por empresas do setor florestal, a máxima precisão na coleta dos dados é visada, pois pode envolver extensas áreas e alto capital investido (SILVA, 2017). Desse modo, de acordo com a finalidade do uso dos dados, deve-se ponderar o erro resultante do emprego dos aplicativos aqui testados. Contudo, ainda que estes não sejam substitutos de equipamentos profissionais precisos para medição de altura, coloca-se como alternativa, levando em conta, também, o baixo custo, praticidade, e situações diversas para seu emprego.

A medição de distância, apenas os tratamentos $T 4 b$ e T6b, em árvores isoladas, não apresentaram diferença significativa a 5\%. Quanto ao plantio, para esta variável, todos apresentaram diferença estatística (Tabela 4). Não foi possível avaliar o aplicativo Smart Distance (T8b), devido a limitação que este apresentou na coleta de dados de acordo com a altura e distância visadas. O tratamento $\mathrm{T} 2 \mathrm{~b}$ não proporcionou funcionamento a partir de determinada distância, impossibilitando a medição desta variável por meio desse método.

Tabela 4. Teste $t$ de Student com $5 \%$ de significância em relação a testemunha distância (TOb).

\begin{tabular}{|c|c|c|c|}
\hline \multirow{2}{*}{ Tratamento } & \multirow{2}{*}{ Método } & \multicolumn{2}{|c|}{ Teste $t$} \\
\hline & & Árvores isoladas & Plantio de Eucalyptus spp. \\
\hline $\mathrm{T} 1 \mathrm{~b}$ & Smart Measure & $-2,33^{*}$ & $9,43 * *$ \\
\hline $\mathrm{T} 2 \mathrm{~b}$ & sMeasure & - & - \\
\hline $\mathrm{T} 3 \mathrm{~b}$ & Distance meter & $-3,56 * *$ & $5,07 * *$ \\
\hline $\mathrm{T} 4 \mathrm{~b}$ & Hypsometer & $-1,54^{\text {ns }}$ & $2,38^{*}$ \\
\hline $\mathrm{T} 5 \mathrm{~b}$ & 3D-Prumo & $-2,77^{*}$ & $-2,84 * *$ \\
\hline T6b & Height and Distance & $-1,56^{\mathrm{ns}}$ & $6,2^{* *}$ \\
\hline $\mathrm{T} 7 \mathrm{~b}$ & Easy Measure & $7,12 * *$ & $8,29 * *$ \\
\hline $\mathrm{T} 8 \mathrm{~b}$ & Smart Distance & - & - \\
\hline
\end{tabular}

$\mathrm{ns}=$ não significativo com $95 \%$ de probabilidade; * = coeficiente significativo a $5 \%$ de probabilidade $(\mathrm{p}<0,05) ; \mathrm{e}^{* *}=$ coeficiente significativo a $1 \%$ de probabilidade $(p<0,01)$.

O cálculo de erros dos métodos testados em árvores isoladas para a variável distância mostrou um menor erro para o tratamento T4b (2,28 m). Mesmo sendo constatada diferença estatística em relação à testemunha, o aplicativo
Distance meter apresentou o menor erro no plantio de Eucalyptus sp., seguido do tratamento T1b, com RMSE de 2,14 m (Tabela 5). 
Tabela 5. Análise de erros para diferentes métodos na medição de distância em árvores isoladas e plantio de Eucalyptus sp.

\begin{tabular}{|c|c|c|c|c|c|c|c|}
\hline & Tratamento & Método & $M E(m)$ & MSE (m) & RMSE (m) & RRMSE (\%) & Erro (\%) \\
\hline \multirow{8}{*}{ Árvores isoladas } & $\mathrm{T} 1 \mathrm{~b}$ & Smart Measure & $-1,02$ & 6,58 & 2,57 & 25,65 & $-10,2$ \\
\hline & $\mathrm{T} 2 \mathrm{~b}$ & sMeasure & - & - & - & - & - \\
\hline & $\mathrm{T} 3 \mathrm{~b}$ & Distance meter & $-1,27$ & 5,27 & 2,3 & 22,96 & $-12,7$ \\
\hline & $\mathrm{T} 4 \mathrm{~b}$ & Hypsometer & $-0,63$ & 5,21 & 2,28 & 22,83 & $-6,28$ \\
\hline & T5b & 3D-Prumo & $-1,77$ & 14,96 & 3,87 & 38,67 & $-17,7$ \\
\hline & $\mathrm{T} 6 \mathrm{~b}$ & Height and Distance & $-0,94$ & 11,29 & 3,36 & 33,60 & $-9,35$ \\
\hline & $\mathrm{T} 7 \mathrm{~b}$ & Easy Measure & 2,82 & 12,53 & 3,54 & 35,39 & 28,2 \\
\hline & $\mathrm{T} 8 \mathrm{~b}$ & Smart Distance & - & - & - & - & - \\
\hline \multirow{8}{*}{ Plantio de Eucalyptus spp. } & $\mathrm{T} 1 \mathrm{~b}$ & Smart Measure & 1,86 & 4,59 & 2,14 & 14,3 & 12,4 \\
\hline & $\mathrm{T} 2 \mathrm{~b}$ & sMeasure & - & - & - & - & - \\
\hline & $\mathrm{T} 3 \mathrm{~b}$ & Distance meter & 1,43 & 4,37 & 2,09 & 13,9 & 9,6 \\
\hline & $\mathrm{T} 4 \mathrm{~b}$ & Hypsometer & 1,32 & 10,66 & 3,27 & 21,8 & 8,8 \\
\hline & $T 5 b$ & 3D-Prumo & $-1,17$ & 6,29 & 2,51 & 16,7 & $-7,8$ \\
\hline & T6b & Height and Distance & 2,09 & 7,64 & 2,76 & 18,4 & 13,9 \\
\hline & $\mathrm{T} 7 \mathrm{~b}$ & Easy Measure & 3,82 & 20,73 & 4,55 & 30,4 & 25,5 \\
\hline & $\mathrm{T} 8 \mathrm{~b}$ & Smart Distance & - & - & - & - & - \\
\hline
\end{tabular}

\section{CONCLUSÃO}

O teste aplicado proporcionou concluir que os aplicativos Smart Measure, Distance meter, Hypsometer, 3D-Prumo e Height and Distance podem ser utilizados para medir alturas em árvores isoladas e o sMeasure, Distance meter, Hypsometer e 3D-Prumo foram satisfatórios em relação ao equipamento tradicionalmente aplicado nos inventários florestais para a variável altura total em plantio de Eucalyptus spp. Já para distância, apenas os aplicativos Hypsometer e Height and Distance são indicados para medir distâncias em árvores isoladas.

Apesar da existência de aplicativos para smartphones, os quais são alternativas para levantamentos dendrométricos em inventários florestais, são poucos os estudos que os analisam em diferentes condições. Desse modo, é necessário e sugere-se que sejam realizados estudos com estes e outros métodos com o uso de aplicativos e softwares disponíveis para smartphones, tais como TRESTIMA $^{\mathrm{TM}}$, Theodolite Droid, Measure Height, Tree Meter, entre outros. Além disso, recomenda-se ainda, testar aplicativos comparando seu desempenho em diferentes tipos de smartphones, e ainda utilizar como testemunha a altura real de árvore abatida.

Em suma, com o presente trabalho conclui-se que a incorporação de novas tecnologias, através de aplicativos para smartphones a custo reduzido, já é uma alternativa para uso em inventários florestais e sua determinação das alturas. Já para distâncias, deve-se ter maior cautela.

\section{REFERÊNCIAS}

ARAÚJO, E.J.G. et al. Relação hipsométrica para candeia (Eremanthus erythropappus) com diferentes espaçamentos de plantio em Minas Gerais, Brasil. Pesquisa Florestal Brasileira, v.32, n.71, p.257-268, 2012.

BIJAK, S.; SARZYŃSKI, J. Accuracy of smartphone applications in the field measurements of tree height. Folia Forestalia Polonica, v.57, n.4, p.240-244, 2015.

BRITO NETO, R.L. et al. Utilização de aplicativo telemóvel para medição da altura total de árvores. In: IV Semana de Engenharia Florestal da Bahia e I Mostra da Pós-graduação em Ciências Florestais da UESB, 2016, Vitória da Conquista. Anais... Bahia: UESB, 2016.

CAMPOS, J.C.C.; LEITE, H.G. Mensuração florestal: perguntas e respostas. 3 ed. Viçosa: UFV, 2009.

CESARO, A. et al. Comparação dos métodos de amostragem de área fixa, relascopia, e de seis árvores, quanto a eficiência, no inventário florestal de um povoamento de Pinus $s p$. Ciência Florestal, v.4, n.1, p.98-108, 1994.

EMBRAPA - Empresa Brasileira de Pesquisa Agropecuária. Atlas climático da região Sul do Brasil: estados do Paraná, Santa Catarina e Rio Grande do Sul. 2.ed. Brasília: Embrapa, 2012.

FAO - Food and Agriculture Organization of the United Nations. Global Forest Resources Assessment: desk reference. Roma: FAO, 2015. Disponível em: http://www.fao.org/forest-resourcesassessment/en/.

GOMIDE, L.R.; LINGNAU, C. Simulação espacial de uma paisagem sob o efeito borda. Floresta, v.39, n.2, p.441-455, 2009. 
IBÁ - Indústria Brasileira de Árvores. Relatório 2017, 2017. Disponível

em: https://iba.org/images/shared/Biblioteca/IBA RelatorioAnual20 17.pdf

IFPR - Instituto Florestal do Paraná. Manual de Inventário Florestal, 2016. Disponível em: http://www.florestasparana.pr.gov.br/arquivos/File/EDITAL/CO NCESSAO/2016/IFPR.CONCESSAO.013.2016/11 MANUAL INVE NTARIO.pdf

KÖPPEN, W.; GEIGER, R. Klimate der Erde. Gotha: Verlag Justus Perthes, 1928.

MARTINI, A. et al. A periodicidade diária do índice de conforto térmico na arborização de ruas de Curitiba-PR. Scientia Plena, v.9, n.5, p.1-9, 2013.

SANQUETTA, R.S. et al. Inventários florestais: planejamento e execução. 3.ed. Curitiba: Multi-Grafic, 2014.

SILVA, G.F. et al. Avaliação de métodos de medição de altura em floretas naturais. Árvore, v.36, n.2, p.341-348, 2012.

SILVA, S.A. Proposta metodológica para auditoria em inventário florestal com enfoque na avaliação de erros de medição. 2017. 87p. (Dissertação de mestrado).

THIERSCH, C.R. et al. Estimativa da relação hipsométrica em clones de Eucalyptus sp. com o modelo de Curtis ajustado por métodos bayesianos empíricos. Árvore, v.37, n.1, p.1-8, 2013.

TOMPPO, E. et al. National Forest Inventories: pathway for common reporting. Heideberg: Springer, 2010.

URBANO, E.; PEREIRA, P.R.; CACAU, F.V.P. Comparação de diferentes equipamentos para medição da altura de árvores em plantio de eucalipto. Atualidades em mensuração florestal. Curitiba: UFPR, 2014.

VASTARANTA, M. et al. Evaluation of a smartphone app for forest sample plot measurements. Forests, v.6, n.4, p.1179-1194, 2015. 